\title{
Gate-Defined Josephson Junctions in Magic-Angle Twisted Bilayer Graphene
}

\author{
Folkert K. de Vries,, , | Elías Portolés,${ }^{1}$ Giulia Zheng, ${ }^{1}$ Takashi Taniguchi, ${ }^{2}$ \\ Kenji Wantanabe, ${ }^{3}$ Thomas Ihn, ${ }^{1}$ Klaus Ensslin, ${ }^{1}$ and Peter Rickhaus ${ }^{1, \dagger}$ \\ ${ }^{1}$ Solid State Physics Laboratory, ETH Zurich, CH-8093 Zurich, Switzerland \\ ${ }^{2}$ International Center for Materials Nanoarchitectonics, \\ National Institute for Materials Science, 1-1 Namiki, Tsukuba 305-0044, Japan \\ ${ }^{3}$ Research Center for Functional Materials, National Institute for Materials Science, 1-1 Namiki, Tsukuba 305-0044, Japan
}

In the past two years, magic-angle twisted bilayer graphene ${ }^{1 / 4}$ has emerged as a uniquely versatile experimental platform that combines metallic, superconducting, magnetic and insulating phases in a single crysta ${ }^{5}[10$. In particular the ability to tune the superconducting state with a gate voltage opened up intriguing prospects for novel device functionality. Here we present the first demonstration of a device based on the interplay between two distinct phases in adjustable regions of a single magicangle twisted bilayer graphene crystal. We electrostatically define the superconducting and insulating regions of a Josephson junction and observe tunable DC and AC Josephson effects11112. We show that superconductivity is induced in different electronic bands and describe the junction behaviour in terms of these bands, taking in consideration interface effects as well. Shapiro steps, a hallmark of the AC Josephson effect and therefore the formation of a Josephson junction, are observed. This work is an initial step towards devices where separate gate-defined correlated states are connected in single-crystal nanostructures. We envision applications in superconducting electronics ${ }^{13 \mid 14}$ and quantum information technology $y^{15 / 16}$ as well as in studies exploring the nature of the superconducting state in magic-angle twisted bilayer graphene.

Gate-tunable superconductivity is a topical field of pure and applied physics research 17 , recently boosted by the realization of two-dimensional (2D) superconductors $\frac{18}{18}$. Switching superconductivity electrostatically on and off makes device design more flexible and circumvents interface and fabrication challenges met when working with multi-material nanostructures. Superconductivity in $2 \mathrm{D}$ has been realised for example at the $\mathrm{LaAlO}_{3} / \mathrm{SrTiO}_{3}$ interface ${ }^{19}$, and in van der Waals materials such as $\mathrm{WTe}_{2}{ }^{20121}$. Because of the large charge carrier densities in most of these systems, in-situ electrostatic control is limited, and therefore gate-tunable Josephson junctions (JJs) have been realised only in a few systems ${ }^{19122}$ where tunability is achieved by proximity to another material or by structural confinement.

Magic-angle twisted bilayer graphene (MATBG) is an ideal 2D platform for in-situ gate-tunable superconductivity $\sqrt{5 / 79}$ While there are numerous potential device applications 13 , 16 , progress towards practical implementations has been hindered by the need for well-defined gated regions. Our multilayer gate technology has enabled us to create a device in a single MATBG crystal with electrostatically defined regions displaying distinct quantum phases. This device represents a new technology that can be used to build integrated 2D electronics using correlated states 5 .

Here we report a first step towards superconducting devices based on distinct quantum phases within a single crystal, by engineering a tunable JJ electrostatically defined in MATBG (Fig. 1 1 , b). We confirm the formation of a JJ by observation of both the DC Josephson effect, with the typical hysteresis found for an underdamped JJ13, and the AC Josephson effect, see Figs. 1k,d. For the latter we apply a radio-frequency (rf) voltage excitation $\left(V_{\mathrm{rf}}\right)$, leading to the observation of Shapiro steps ${ }^{12}$, that are proportional to the frequency $f_{\mathrm{rf}}$ and the superconducting flux quantum $h / 2 e$. Furthermore, we demonstrate that the device can be tuned in situ from the short to the long JJ regime, establishing the flexibility of our approach.

The device introduced here has three different gate layers that enable electrostatically defining several JJs (Fig. 1 1 ,b). The global carrier density $n$ in the MATBG can be changed by a graphite bottom gate. A pair of local top-gates (L, $\mathrm{R}$ ) controls the density of the leads $n_{\ell}$ (when tuned together with the bottom gate) and defines a junction of length $L_{\mathrm{j}}=100 \mathrm{~nm}$ and width $W=2.3 \mu \mathrm{m}$. The density in this junction $n_{\mathrm{j}}$ is gated by gate C. In order to form JJs with different lengths $L_{\mathrm{j}}$ we tune another gate or a combination of gates out of the superconducting state, which changes the regions of the device corresponding to densities $n_{\ell}$ and $n_{\mathrm{j}}$ accordingly. All measurements are performed at a temperature $T=25 \mathrm{mK}$ (unless stated otherwise) in a two-terminal setup, where we apply a current $I$ and measure the voltage $V$, from which the differential resistance $R$ is calculated. We correct for contact and lead resistances in the $I-V$ linetraces, but not in $R$ colour maps. More details on the device fabrication and measurement setup are presented in Methods and Supplementary Information (SI), Sec. I.

Before returning to the JJ we introduce the energy bands and the bulk superconducting state in our MATBG device. In Fig. $2 a$ we show $R$ as a function of carrier density $n$. Peaks appear in $R$ at the charge-neutrality point 

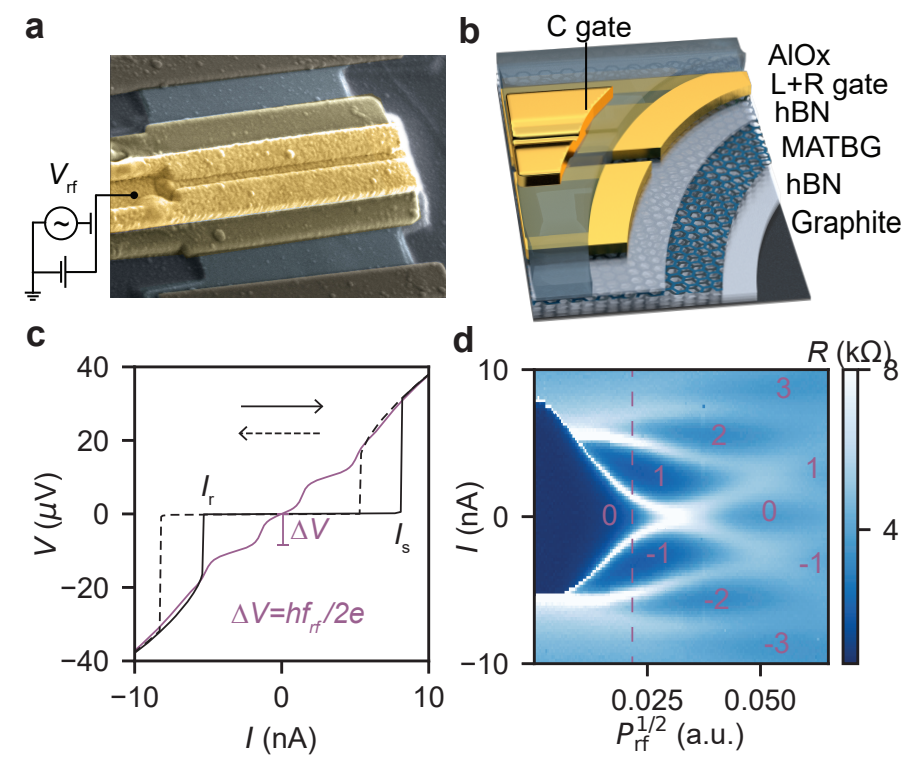

FIG. 1. DC and AC Josephson effect in a MATBG gate-defined Josephson junction. a, False-coloured, tilted scanning electron microscopy image of a similar device and $\mathbf{b}$, cross-section diagram of the device. A $100 \mathrm{~nm}-\mathrm{long}$ JJ is formed by tuning the MATBG into the superconducting state with the graphite bottom-gate and left (L) and right (R) top-gate electrodes, while the center (C) gate tunes the MATBG locally into a non-superconducting state. The gate electrodes are separated from the MATBG by insulating hexagonal boron nitride (hBN), and from each other by an aluminium-oxide (AlOx) layer. c, The applied current $I$ and measured voltage $V$ (black) reveal a switching and retrapping current $\left(I_{\mathrm{s}}\right.$ and $\left.I_{\mathrm{r}}\right)$ at density $n_{\mathrm{j}}=-4.6 \times 10^{12} \mathrm{~cm}^{-2}$ in the junction area. Applying a voltage drive $\left(V_{\mathrm{rf}}\right)$ at frequency $f_{\mathrm{rf}}=5 \mathrm{GHz}$ to gate C induces Shapiro steps (violet). d, differential resistance $R$ as a function of $I$ and the square root of power $\left(P_{\mathrm{rf}}^{1 / 2}\right)$, showing the Shapiro step spectrum of the JJ. The step number is indicated, as is the trace plotted in $\mathbf{c}$.

$(\mathrm{CNP})$, and at full $\left(n_{\mathrm{s}}\right)$ and half-filling of the moiré bands, corresponding to a twist angle of $\theta=1.06^{\circ}$. Based on this observation we sketch the energy bands and gaps ${ }^{14}$ (inset Fig. 2 a), containing the $1^{\text {st }}$ moiré bands (blue and green), commonly referred to as the flat bands, with correlated insulator gaps at $n_{\mathrm{s}} / 2^{6 \text { 699. The }} 1^{\text {st }}$ band is separated from the dispersive $2^{\text {nd }}$ and $3^{\text {rd }}$ moiré bands (violet) by a band gap. Note that the $2^{\text {nd }}$ and $3^{\text {rd }}$ band are separated in energy, which follows from measurements obtained in the JJ-device configuration (Fig $3 \mathrm{a}, \mathrm{b})$, but contradicts existing band-structure calculations ${ }^{14}$. To reveal the superconducting behaviour of the device we measure the differential resistance $R$ as a function of current $I$ and density $n$ (Fig. $2 \mathrm{~b}$ ). We observe plateaus and the typical peak in $R$ at critical supercurrent $I_{\mathrm{c}}$, where the device switches out of the bulk superconducting state. The largest $I_{\mathrm{c}}$ is observed at $n=-1.5 \times 10^{12} \mathrm{~cm}^{-2}$ in the superconducting dome around $-n_{\mathrm{s}} / 2$. Around $+n_{\mathrm{s}} / 2$ a smaller superconducting dome is observed. This in accordance with earlier reported phase diagrams for MATBG of $\theta=1.06$ 听7 . We estimate a superconducting coherence length $\xi=70 \mathrm{~nm}$ from $I_{\mathrm{c}}$ measurements as a function of temperature $T$ and perpendicular magnetic field $B_{\perp}$ (SI Sec. III).

Having established superconductivity in the MATBG device, we describe the effect of detuning $n_{\mathrm{j}}$ while keeping $n_{\ell}$ superconducting. Figure $3 \mathrm{a}, \mathrm{b}$ shows $R$ at fixed $I$ (top panels) as well as its dependence on $I$ (bottom panels) as a function of $n_{\mathrm{j}}$, while $n_{\ell}$ is close to $-n_{\mathrm{s}} / 2$ (a) or to $+n_{\mathrm{s}} / 2$ (b), respectively. As the quasiparticle current and the capacitance between the contacts influences the maximum supercurrent ${ }^{13}$, we refer to this as the 'switching current' $I_{\mathrm{s}}$ from now on. Comparing the measurements to the bulk behaviour shown in Fig. $2 \mathrm{~b}$, we still observe a finite $I_{\mathrm{s}}$ when $n_{\mathrm{j}}$ is tuned out of the superconducting state. That is, we observe the DC Josephson effect. Note that when $n_{\mathrm{j}}=n_{\ell}$ the entire device is superconducting (shaded regions in Figs. 3p,d). In Fig. 33, b the JJ length $L_{\mathrm{j}}=100 \mathrm{~nm}$ is on the order of the estimated $\xi=70 \mathrm{~nm}$, indicative of the short JJ regime ${ }^{1323}$. Also, for the JJ with $n_{\ell}=-1.5 \times 10^{12} \mathrm{~cm}^{-2}$ we observe signatures of ballistic transport ${ }^{24}$ (SI Sec. III); $I_{\mathrm{s}}$ is thus expected to be limited by the interfaces. In addition to the JJs with $L_{\mathrm{j}}=100 \mathrm{~nm}$, our device allows us to realize other lengths, by using combinations of gates L $(650 \mathrm{~nm})$, $\mathrm{R}(650 \mathrm{~nm})$ and $\mathrm{C}(100 \mathrm{~nm})$. Overall, $I_{\mathrm{s}}$ as a function of $n_{\mathrm{j}}$ is reduced for increased $L_{\mathrm{j}}$, as presented in Fig. 3c,d, and in the inset of of Fig. 3p, consistent with $L_{\mathrm{j}} \gg \xi$ for the $L_{\mathrm{j}}=650 \mathrm{~nm}$ and $1400 \mathrm{~nm}$ JJs, which means that these junctions are in the long-JJ regime ${ }^{13 / 23}$ where the transport in the junction area is limiting $I_{\mathrm{S}}$.

By changing $n_{\mathrm{j}}$ we tune the JJs through the energy bands depicted in Fig. $2 \mathrm{a}$, as reflected in the $R$ trace in Fig. $3 \mathrm{p}, \mathrm{b}$ (highlighted with corresponding background colours). We now analyse how $I_{\mathrm{s}}$ changes with $n_{\mathrm{j}}$, discuss this in terms 


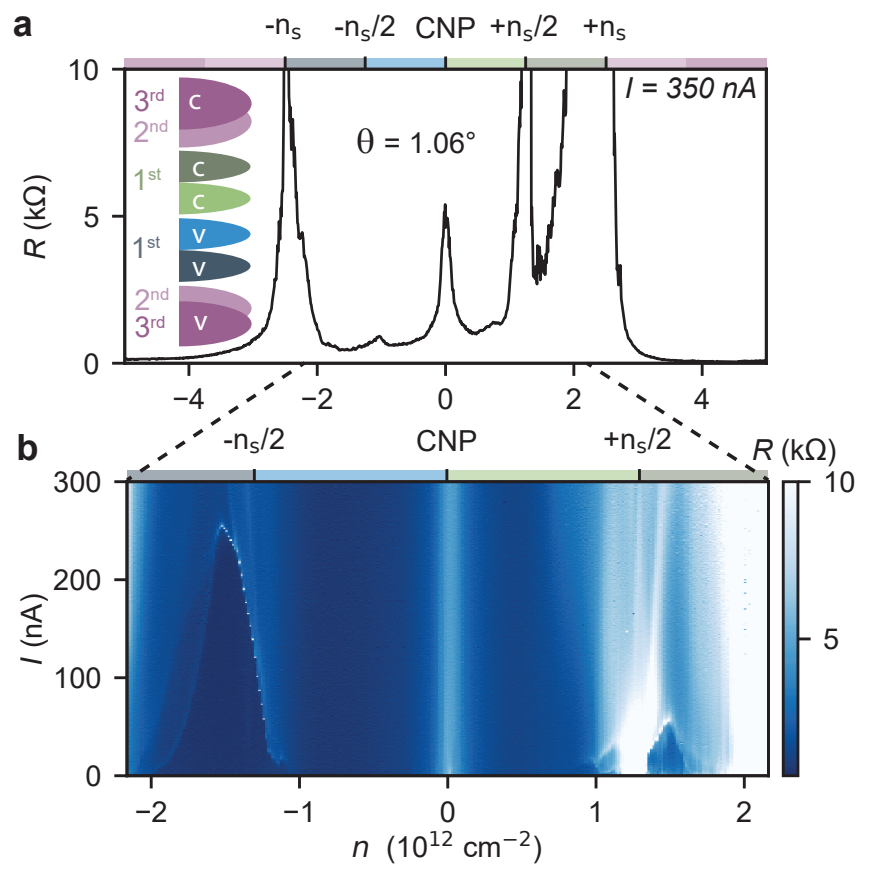

FIG. 2. Bulk superconductivity in MATBG. a $R$ as a function of $n$ shows pronounced peaks for $\pm n_{\mathrm{s}}$ and $\pm n_{\mathrm{s}} / 2$, symmetrically around the CNP. The inset shows a schematic representation of the energy bands in MATBG, where the flat valence (v) and conduction (c) bands (blue and green) and higher moiré bands (violet) are indicated. The colours on top of the panels indicate the relation between the density and the bands. $\mathbf{b} R$ as a function of $I$ and $n$ reveals superconductivity around $\pm n_{\mathrm{s}} / 2$.

of the junction area ( $\mathrm{N}$ area) and the interfaces between the leads and the junction (N-S interfaces). We observe step-like reductions in $I_{\mathrm{S}}$ when $n_{\mathrm{j}}$ crosses over to another band and a more gradual change of $I_{\mathrm{S}}$ within a given band (Fig. 3a,b). These steps in $I_{\mathrm{s}}$ occur when the Fermi level crosses a band gap at the CNP and at $n_{\mathrm{j}}= \pm n_{\mathrm{s}}$, and a correlated insulator gap at $n= \pm n_{\mathrm{S}} / 2$, which reduces the transparency of the $\mathrm{N}-\mathrm{S}$ interfaces. We note that the abrupt changes can also have a contribution from differences between the eigenstates on each side of the interface, such as topological indices $\frac{10}{}$ or valley/spin flavours 25 . Schottky barriers and interfacial defects can be neglected as the S-N interfaces are created in a single crystal. Regarding the gradual change of $I_{\mathrm{S}}$ within a band, we suspect the Fermi velocity $v_{\mathrm{F}}$ to be most important for the proximity effect. BCS theory predicts $\xi$ in the junction area to scale linearly with $v_{\mathrm{F}}$ of the carriers in the $\mathrm{N}$ area ${ }^{13}$. Also, the $\mathrm{N}-\mathrm{S}$ interface transparency is affected by a mismatch between $v_{\mathrm{F}}$ in the leads and in the $\mathrm{N}$ are ${ }^{26}$, respectively. The dramatic changes in $v_{\mathrm{F}}$ with $n$ in the bands of MATBG1 14 therefore impacts the induced superconductivity. Note that $L_{\mathrm{j}}$ slightly changes due to the gate geometry used. For increasing $\left|n_{\mathrm{j}}-n_{\ell}\right|, L_{\mathrm{j}}$ could increase by approximately $20 \mathrm{~nm}$ (SI Sec. II).

In the $1^{\text {st }}$ moiré bands (blue and green) we observe rather large $I_{\mathrm{s}}$ values, in line with the weakly insulating states at $\pm n_{\mathrm{s}} / 2$ and the CNP, leading to a significant $I_{\mathrm{s}}$ even if the junction is $1400 \mathrm{~nm}$ long (Fig. 3r). For $L_{\mathrm{j}}=650 \mathrm{~nm}$ we observe a non-monotonic behaviour of $I_{\mathrm{s}}$ between $-n_{\mathrm{s}} / 2$ and the CNP (red arrow in Fig. 35)). This could be due to an optimum in $v_{\mathrm{F}}$, or might be caused by electron correlations that are already present, yet not strong enough to cause a superconducting ground state in our device ${ }^{9}$.

An interesting JJ is realized once $n_{\mathrm{j}}$ is tuned to the opposite superconducting dome. The switching current $I_{\mathrm{S}}$ is increased around $-n_{\mathrm{s}} / 2$ for all three $L_{\mathrm{j}}$ values as shown in Fig. 3p,d, as well as for $L_{\mathrm{j}}=650 \mathrm{~nm}$ and $1400 \mathrm{~nm}$ as shown in Fig. 3r. If the leads and junction in this $\mathrm{S}-\mathrm{S}-\mathrm{S}$ configuration exhibit the same superconducting state, $I_{\mathrm{s}}$ would solely depend on the interfaces and not on $L_{\mathrm{j}}$. We observe, however, a dependence on $L_{\mathrm{j}}$ in both Fig. 35 and $\mathrm{d}$, suggesting that the superconductivity in the two domes differ. Such a scenario is an interesting avenue for future research, as it could be possible that we formed a JJ with two distinct superconductors.

When we tune $n_{\mathrm{j}}$ into the $2^{\text {nd }}$ and $3^{\text {rd }}$ moiré band (violet colours in Fig. 2a), $I_{\mathrm{s}}$ in Fig. $3(\mathrm{a}, \mathrm{b})$ is greatly reduced, due to a crossing of the gap $\left( \pm n_{\mathrm{s}}\right)$ at the N-S interfaces. In addition, another step in $R$ is observed for both the valence bands in Fig. 33, as well as for the conduction bands in Fig. 3p. At the same time, $I_{\mathrm{s}}$ is either decreased or even reduced to zero, respectively. Based on this observation and supported by Fabry-Pérot oscillations (SI Sec. III), we infer that we observe the band edge of the $3^{\text {rd }}$ moiré band, and this band is therefore offset in energy with respect to the $2^{\text {nd }}$ 

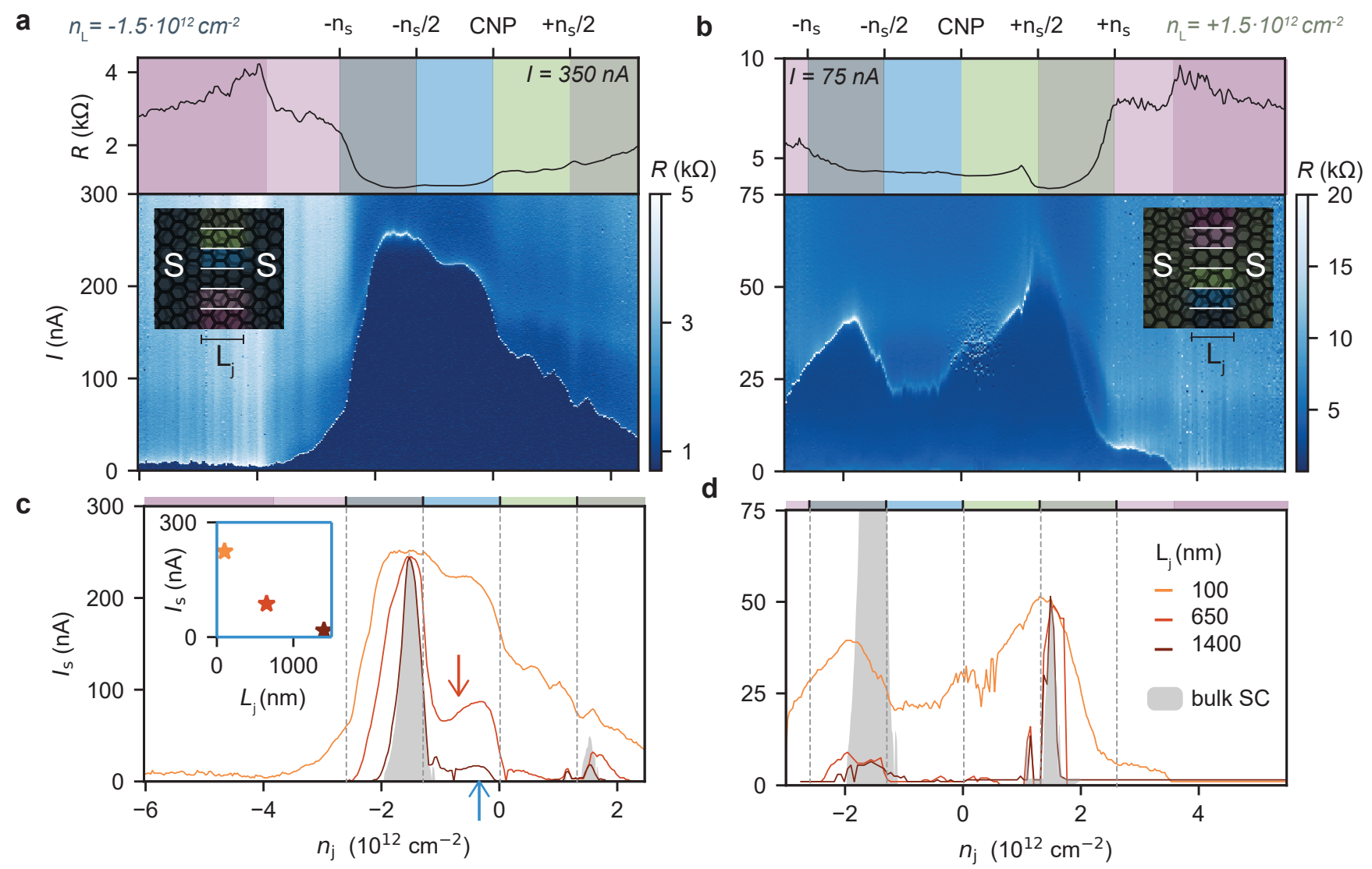

FIG. 3. Gate-defined and tunable Josephson junctions. a,b, Gate-defined JJs with $L_{\mathrm{j}}=100 \mathrm{~nm}$ and $n_{\ell}$ tuned to either the negatively (a) or positively doped (b) superconducting domes as indicated on the top axis. The line trace, taken at the $I$ value indicated, and colour-map plot $R$ as a function of $n_{\mathrm{j}}$ (and $I$ ). Overlaid are the colours indicating of energy bands sketched in Fig. 1 1 a. c,d, $I_{\mathrm{s}}$ for different $L_{\mathrm{j}}$ as a function of $n_{\mathrm{j}}$. Bulk $I_{\mathrm{c}}$ versus $n$ is plotted for reference (grey). The inset displays $I_{\mathrm{s}}$ versus $L_{\mathrm{j}}$ for $n_{\mathrm{j}}$ indicated by the blue arrow.

moiré band (Fig. 2 a). While before we connected the abrupt changes of $R$ and $I_{\mathrm{s}}$ to the Fermi level crossing a gap, this is unlikely to be the case here, as we do not observe a resistance peak in Fig 2 around $n=-4.6 \times 10^{12} \mathrm{~cm}^{-2}$. We speculate that the transition could occur because of a sudden change in $v_{\mathrm{F}}$ (mismatch) or a mismatch in eigenstates in the leads and the junction.

We further studied this $n_{\mathrm{j}}$-regime for the $100 \mathrm{~nm} \mathrm{JJ}$ with $n_{\ell}=-1.5 \times 10^{12} \mathrm{~cm}^{-2}$ by measuring the AC Josephson effect. As $n_{\mathrm{j}}$ is tuned into the $2^{\text {nd }}$ and $3^{\text {rd }}$ moiré band, where the $\mathrm{N}-\mathrm{S}$ interfaces are less transparent, we observe the AC Josephson effect in the form of Shapiro steps $\frac{12}{\sqrt{12}}$. In the $3^{\text {rd }}$ moiré band $\left(n_{\mathrm{j}}=-4.6 \times 10^{12} \mathrm{~cm}^{-2}\right)$ the steps have the trivial height of $\Delta V=(h / 2 e) \times f_{\mathrm{rf}}$ for both frequencies of $f_{\mathrm{rf}}=5 \mathrm{Ghz}$ and $1.81 \mathrm{Ghz}$, see Fig. 1 1 ,d, and Fig. 4a, respectively, and SI Sec. IV. Also, no integer steps are missing or only occurring at a specific $P_{\mathrm{rf}}^{1 / 2}$, as could

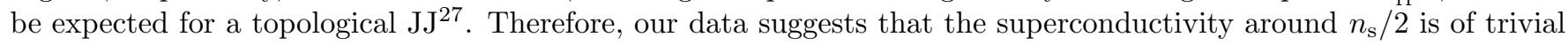
rather than of topological nature.

The Shapiro-step spectrum for $n_{\mathrm{j}}$ tuned in the $2^{\text {nd }}$ moiré band only $\left(n_{\mathrm{j}}=-3.5 \times 10^{12} \mathrm{~cm}^{-2}\right)$ in Fig. 4 b shows a dependence on $P_{\mathrm{rf}}^{1 / 2}$ very different from that in Fig. 4 . The steps are less dense and fade more quickly with respect to $P_{\mathrm{rf}}^{1 / 2}$. We attribute the fading to thermal and impurity smearing of $R$, impacting the lower-valued $R$-peaks in Fig. 40 more than in Fig. 4a. In addition, the hysteresis $\left(I_{\mathrm{S}} / I_{\mathrm{r}}\right)$ differs, suggesting a change in the JJ that can be traced back to the onset of the $3^{\text {rd }}$ moiré band as the $n_{\mathrm{j}}$-dependence in Fig. 4 p reveals.

We compare the trend of this JJ behaviour to simulations using the resistively and capacitively shunted junction mode 13 (see Methods for details). In this model, the resistance $R_{\mathrm{j}}$, allowing for the flow of normal current, and $C_{\mathrm{j}}$, the capacitance between the leads, are both connected in parallel to an ideal JJ with a given $I_{\mathrm{c}}$. Together, these parameters are captured in the Stewart-McCumber parameter $\beta \propto I_{\mathrm{c}} R_{\mathrm{j}}^{2} C_{\mathrm{j}}$. In the inset of Fig. $4 \mathrm{p}$, we show the simulated $I_{\mathrm{s}} / I_{\mathrm{r}}$ as a function of $\beta$, and we estimate $\beta=4.2$ and $\beta=1.5$ for the $I_{\mathrm{s}} / I_{\mathrm{r}}$ from Fig. $4 \mathrm{a}, \mathrm{b}$, respectively. 

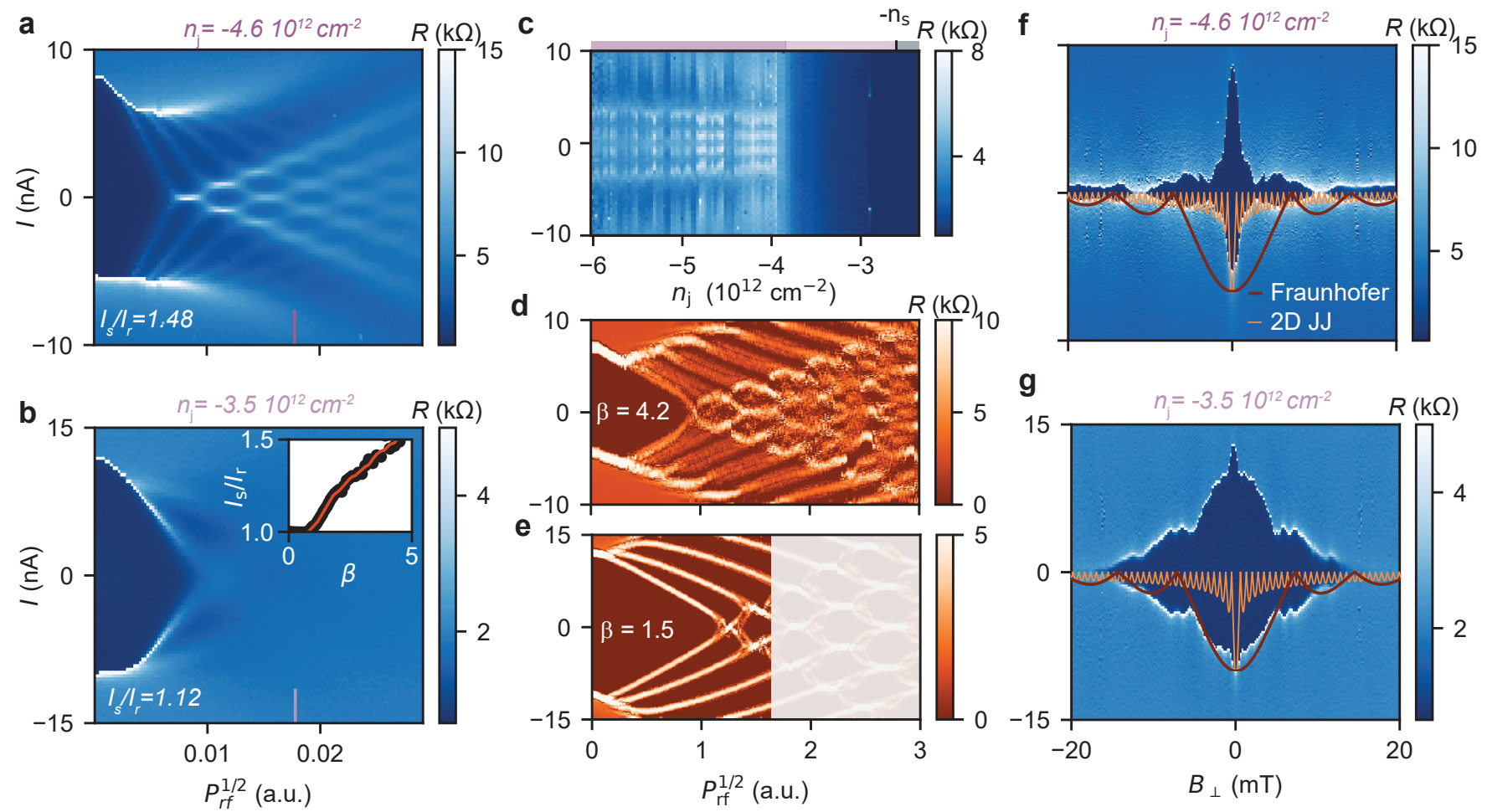

FIG. 4. AC Josephson effects for the $2^{\text {nd }}$ and $\mathbf{3}^{\text {rd }}$ moiré band. $\mathbf{a}, \mathbf{b}$, Measurement of the Shapiro steps, visible in $R$ versus $I$ for different $P_{\mathrm{rf}}^{1 / 2}$, for $n_{\mathrm{j}}$ as indicated. The hysteresis $I_{\mathrm{s}} / I_{\mathrm{r}}$ is given. The inset in $\mathbf{b}$ shows the calculated $I_{\mathrm{s}} / I_{\mathrm{r}}$ for different $\beta$. c, $R$ versus $I$ measured as a function of $n_{\mathrm{j}}$ at $P_{\mathrm{rf}}^{1 / 2}$ indicated in $\mathbf{c}, \mathbf{d}$. The colour bar on top refers to the bands in Fig. 2a. d,e, Shapiro steps simulated using the resistively and capacitively shunted junction (RCSJ) model (see Methods) using the $\beta$ value indicated, $R_{\mathrm{j}}=2.5 \mathrm{k} \Omega$ and $R_{\mathrm{j}}=1.14 \mathrm{k} \Omega$ for the upper and lower panels, respectively, and $C_{\mathrm{j}}=32 \mathrm{fF}$ for both. The shaded area in $\mathbf{e}$ is added for better comparability. f,g, $R$ as a function of $I$ and $B$ shows the magnetic interference pattern of the $100 \mathrm{~nm}$ JJ with $n_{\ell}=-1.5 \times 10^{12} \mathrm{~cm}^{-2}$ for the $n_{\mathrm{j}}$ value indicated. For both scenarios we plotted a theoretical model for a $3 \mathrm{D}$ JJ and for a JJ in a $2 \mathrm{D}$ superconductor.

Then, we simulate Shapiro-step spectra using $\beta$, the measured $I_{\mathrm{s}}$, a constant $C_{\mathrm{j}}$ (the device geometry is constant), and we choose $R_{\mathrm{j}}=2.5 \mathrm{k} \Omega$ based on the normal-state resistance for $\beta=4.2$, of which $R_{\mathrm{j}}=1.14 \mathrm{k} \Omega$ for $\beta=1.5$ follows from the earlier-mentioned constraint on $C_{\mathrm{j}}$. The simulated spectra in Fig. A4 qualitatively reproduce the measured spectra of Fig. 4a,b. As the smearing is not captured in the model, we added a semi-transparent box in Fig. 伴 for better comparability. The increase of $R_{\mathrm{j}}$ when entering the $3^{\text {rd }}$ moiré band means that the quasiparticle transport through the junction is reduced, which can be important for future device applications such as detectors $\$$ 13|14.

Lastly, we report magnetic interference measurements in this $n_{\mathrm{j}}$-regime and compare them to theoretical models (see Methods). In a textbook 3D JJ, applying $B_{\perp}$ results in a modulation of $I_{\mathrm{S}}$ described by a Fraunhofer pattern with the periodicity $\Delta B_{\perp}=h / 2 e /\left(L_{\mathrm{j}} W\right)$, and $I_{\mathrm{s}}$-maxima that scale with $1 / B_{\perp} \underline{13}$. For a JJ in a $2 \mathrm{D}$ superconductor, $B_{\perp}$ also penetrates the leads, and $\Delta B_{\perp}=1.8 \Phi_{0} / W^{2}$ and the $I_{\mathrm{s}}$-maxima scale with $1 / B_{\perp}^{1 / 228}$. In Fig. 4 . at $n_{\mathrm{j}}=$ $-4.6 \times 10^{12} \mathrm{~cm}^{-2}$, the $I_{\mathrm{s}}$-maxima follow the $1 / B^{1 / 2}$ behaviour, but the periodicity matches neither theoretical pattern. The ill-defined periodicity is likely to be caused by disorder in the junction area and the leads due to twist-angle inhomogeneity $2 \underline{29}$. For the interference pattern shown in Fig. $4 \mathrm{~s}$, measured at $n_{\mathrm{j}}=-3.48 \times 10^{12} \mathrm{~cm}^{-2}, I_{\mathrm{s}}$ is closer to the $1 / B_{\perp}$ scaling of a Fraunhofer pattern. However, it also lacks a clear periodicity, even though the smaller $I_{\mathrm{S}}$ oscillations on the larger background compare better to the 2D JJ pattern. Although we find clearly different interference patterns, further theoretical and experimental work is needed to understand the patterns themselves.

In conclusion, we have shown a proof-of-principle gate-defined superconducting device in MATBG, and explored this JJ by tuning through the different moiré bands. We defined JJs with different lengths and different superconducting states in a single device, establishing the flexibility of our approach. This work enables future technological applications, by virtue of its planar and gate-tunable superconductivity ${ }^{15 / 16}$, as well as future investigations of the nature of the superconducting state in MATBG, for example by fabricating superconducting quantum interference devices or by creating JJs with a ferromagnetic barrier. 


\section{METHODS}

\section{Device Fabrication}

We assemble the heterostructure using the dry pick-up method ${ }^{30}$. As a first step, a large $(>40 \mu \mathrm{m} \times 40 \mu \mathrm{m})$ mechanically exfoliated graphene flake is identified on a p:Si/ $\mathrm{SiO}_{2}(285 \mathrm{~nm})$ wafer. The graphene flake is scratched into two pieces using a sharp $(2.5 \mathrm{\mu m}$ tip diameter) tungsten needle which is controlled by a micromanipulator. Using a PDMS/PC stamp, the top hBN layer $(27 \mathrm{~nm})$ is picked up at a $T=80^{\circ} \mathrm{C}$. At $40{ }^{\circ} \mathrm{C}$, we pick up the first graphene piece, rotate the stage by $1.1^{\circ}$ and pick up the second piece. The twisted graphene is encapsulated with a bottom $\mathrm{hBN}$ flake $(24 \mathrm{~nm})$, the flake is in contact with the stack at $40^{\circ} \mathrm{C}$ and picked up at $80^{\circ} \mathrm{C}$. At the same temperature, the graphite layer is picked up. PC is detached from PDMS at $150^{\circ} \mathrm{C}$ and removed in dichloromethane. The top and bottom $\mathrm{hBN}$ thicknesses are determined using atomic force microscopy (AFM). AFM images serve to identify clean and bubble-free regions of the stack. Next, we define $1 \mathrm{D}$ contacts ${ }^{31}$ to graphene by electron beam lithography (EBL), reactive ion etching $\left(\mathrm{RIE}, \mathrm{CHF}_{3} / \mathrm{O}_{2}, 40 / 4 \mathrm{sccm}, 60 \mathrm{~W}\right.$, with a $0.6 \mathrm{~nm} / \mathrm{s} \mathrm{hBN}$ etching rate, for $68 \mathrm{~s}$ ) and electron beam evaporation $(\mathrm{Cr} / \mathrm{Au}, 10 / 70 \mathrm{~nm})$. Then, top-gates are defined by EBL $(\mathrm{Cr} / \mathrm{Au}, 10 / 60 \mathrm{~nm})$ and the graphene is etched (RIE, $\mathrm{CHF}_{3} / \mathrm{O}_{2}, 40 / 4 \mathrm{sccm}, 60 \mathrm{~W}, 120 \mathrm{~s}$, followed by $\mathrm{O}_{2}, 40 \mathrm{sccm}, 60 \mathrm{~W}, 10 \mathrm{~s}$ ). Using atomic layer deposition (ALD), $30 \mathrm{~nm}$ of $\mathrm{AlO}_{x}$ are deposited at $T=150^{\circ} \mathrm{C}$. In a final step, we define the central gates by EBL and deposit $\mathrm{Cr} / \mathrm{Au}$, $10 / 110 \mathrm{~nm}$.

\section{Measurement setup}

We use a DC voltage source built in-house with a $10 \mathrm{M} \Omega$ connected in series to generate the bias current, amplify the voltage using a voltage amplifier built in-house and measure it with an Hewlett Packard 34401A multimeter. We measure in a 2 terminal setup, and subtracted a series resistance of $785 \Omega$ in Fig. 1 $1 \mathrm{p}$. The bottom gate, $\mathrm{L}$ gate and $\mathrm{R}$ gate are each connected to a $\mathrm{DC}$ voltage source. The $\mathrm{C}$ gate is connected to a $\mathrm{DC}$ voltage source and, using a bias $\mathrm{T}$ with $R=10 \mathrm{k} \Omega$ and $C=10 \mathrm{nF}$, to a Rohde und Schwarz SMB 100A signal generator.

\section{RCSJ model}

We model our JJ with the resistively and capacitively shunted junction (RCSJ) mode 13 . Replacing the voltage across the junction, $V$, with the superconducting phase between the two electrodes of the junction, $\phi$, using $V=$ $\left(\Phi_{0} / 2 \pi\right) \times d \phi / d t^{11}$, we obtain the second order differential equation with reduced units ${ }^{32}$.

$$
\frac{I}{I_{\mathrm{c}}}=\sin \phi+\frac{d \phi}{d \tilde{\tau}}+\beta \frac{d^{2} \phi}{d^{2} \tilde{\tau}^{2}},
$$

containing the Stewart-McCumber parameter

$$
\beta=\frac{2 \pi R_{\mathrm{j}}^{2} C_{\mathrm{j}} I_{\mathrm{c}}}{\Phi_{0}},
$$

and $\tilde{\tau}=t / \tau_{\mathrm{J}}$ with

$$
\tau_{\mathrm{J}}=\frac{\Phi_{0}}{2 \pi R_{\mathrm{j}} I_{\mathrm{c}}}
$$

being the Josephson time constant.

The resistance $R_{\mathrm{j}}$ depends on the state of the junction. When operating it beyond its critical current, $R_{\mathrm{j}}$ corresponds to $R_{\mathrm{n}}$, the normal state resistance of the junction. When a supercurrent is flowing, $R_{\mathrm{j}}$ accounts for tunnelling of quasiparticles through the junction. The quasiparticle resistance $R_{\mathrm{qp}}$ is, in general, expected to be higher than $R_{\mathrm{n}}$. It is hard to estimate the value of $R_{\mathrm{qp}}$ and its dependence on the current $I$, espacially since our knowledge about the electronic state in the junction area is limited. We have therefore decided to assume $R_{\mathrm{j}}$ is on the order of $R_{\mathrm{n}}$. 


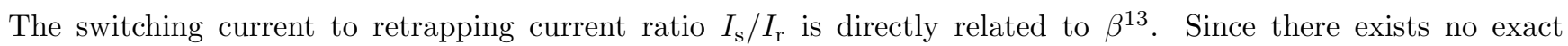
analytical relation between the parameters, we compute it (inset in Fig. $4 \mathrm{~b}$ ). We then obtain $\beta_{\mathrm{C}}=4.2$ and $\beta_{\mathrm{C}}=1.5$ for $n_{\mathrm{j}}=-4.6 \times 10^{12} \mathrm{~cm}^{-2}$ and $n_{\mathrm{j}}=-3.5 \times 10^{12} \mathrm{~cm}^{-2}$ respectively.

For modelling the behavior of an RF-driven Josephson junction we add a sinusoidal term to the current bias term of equation (1), thus obtaining

$$
\frac{I}{I_{\mathrm{c}}}+\frac{I_{\mathrm{RF}}}{I_{\mathrm{c}}} \sin \tilde{\omega} \tilde{\tau}=\sin \phi+\frac{d \phi}{d \tilde{\tau}}+\beta_{\mathrm{C}} \frac{d^{2} \phi}{d^{2} \tilde{\tau}^{2}}
$$

with $\tilde{\omega}=\omega_{\mathrm{RF}} \tau_{\mathrm{J}}$ the reduced angular frequency of the RF-source $\mathrm{e}^{33}$.

In this mathematical treatment we neglect the frequency-dependent coupling of the RF-signal to the junction. Furthermore, we assume the relationship between the sinusoidal voltage of the RF-wave and the resulting AC current through the junction to be linear.

The reduced angular frequency, $\tilde{\omega}$, is the relevant parameter for the observation of the Shapiro steps. Only if the condition $\tilde{\omega} \ll 1$ is fulfilled, the phase of the junction is properly locked to that of the RF-source, and Shapiro steps can be observed 34 . Three parameters determine $\tilde{\omega}$ : The RF-frequency, which is in our simulations always fixed to $\omega_{\mathrm{RF}}=1.81 \mathrm{GHz}$, the critical current of the junction, which we fix to either $I_{\mathrm{C}}=7 \mathrm{nA}$ or $I_{\mathrm{C}}=12 \mathrm{nA}$, and the resistance $R$ of the junction. As we mentioned previously, we extract experimentally $\beta_{\mathrm{C}} \propto I_{\mathrm{C}} C R^{2}$, which leaves us with two free parameters for our modelling, $R$ and $C$. We start at $\beta_{\mathrm{C}}=4.2$ and choose $R=2.5 \mathrm{k} \Omega$ since it is of the order of the normal state resistance for $n_{\mathrm{j}}=-4.6 \times 10^{12} \mathrm{~cm}^{-2}$. We compute the resulting capacitance $C=31.2 \mathrm{fF}$ and perform the simulation $(4 \mathrm{~d})$. When moving to $\beta_{\mathrm{C}}=1.5$, for simulating the $n_{\mathrm{j}}=-3.5 \times 10^{12} \mathrm{~cm}^{-2} \mathrm{case}$, we keep $C$ constant and obtain $R=1.14 \mathrm{k} \Omega$, obtaining the result shown in Fig. 4 .

\section{Magnetic interference patterns}

Here we provide the equations used to plot the theoretical magnetic interference patterns. The Fraunhofer interference pattern follows

$$
I\left(B_{\perp}\right)=I_{\mathrm{c}}\left|\frac{\sin \left(\pi B_{\perp} L_{\mathrm{j}} W / \Phi_{0}\right)}{\pi B_{\perp} L_{\mathrm{j}} W / \Phi_{0}}\right|
$$

For the Josephson junction in a 2D superconductor we use the approximation

$$
I\left(B_{\perp}\right)=0.61 \frac{I_{\mathrm{c}}}{W} \sqrt{\frac{\Phi_{0}}{\left|B_{\perp}\right|}}\left|\cos \left(1.72\left|B_{\perp}\right| W^{2} / \Phi_{0}-\pi / 4\right)\right| .
$$

given in Ref. 28 . 
* devriesf@phys.ethz.ch

† peterri@phys.ethz.ch

[1] Suárez Morell, E., Correa, J. D., Vargas, P., Pacheco, M. \& Barticevic, Z. Flat bands in slightly twisted bilayer graphene: Tight-binding calculations. Phys. Rev. B 82, 121407 (2010).

[2] Bistritzer, R. \& MacDonald, A. Moiré bands in twisted double-layer graphene. Proc. Natl. Acad. Sci. 108, 12233 - 12237 (2011).

[3] Nam, N. N. T. \& Koshino, M. Lattice relaxation and energy band modulation in twisted bilayer graphene. Phys. Rev. B 96, $075311(2017)$.

[4] Guinea, F. \& Walet, N. R. Continuum models for twisted bilayer graphene: Effect of lattice deformation and hopping parameters. Phys. Rev. B 99, 205134 (2019).

[5] Cao, Y. et al. Unconventional superconductivity in magic-angle graphene superlattices. Nature 556, 43-50 (2018).

[6] Cao, Y. et al. Correlated insulator behaviour at half-filling in magic-angle graphene superlattices. Nature 556, 80-84 (2018).

[7] Yankowitz, M. et al. Tuning superconductivity in twisted bilayer graphene. Science 363, 1059-1064 (2019).

[8] Sharpe, A. L. et al. Emergent ferromagnetism near three-quarters filling in twisted bilayer graphene. Science 365, 605-608 (2019).

[9] Lu, X. et al. Superconductors, orbital magnets and correlated states in magic-angle bilayer graphene. Nature 574, 653-657 (2019).

[10] Nuckolls, K. P. et al. Strongly correlated chern insulators in magic-angle twisted bilayer graphene. arXiv preprint arXiv:2007.03810 (2020).

[11] Josephson, B. Possible new effect in superconducting tunneling. Phys. Lett. 1, 251-253 (1962).

[12] Shapiro, S. Josephson currents in superconducting tunneling: The effect of microwaves and other observations. Phys. Rev. Lett. 11, 80-82 (1963).

[13] Tinkham, M. Introduction to superconductivity (Courier Corporation, 2004).

[14] Braginski, A. I. Superconductor electronics: Status and outlook. J. Supercond. Nov. Magn. 32, 23-44 (2019).

[15] Wendin, G. Quantum information processing with superconducting circuits: a review. Rep. Progr. Phys. 80, 106001 (2017).

[16] Liu, X. \& Hersam, M. C. 2d materials for quantum information science. Nat. Rev. Mater. 4, 669-684 (2019).

[17] De Simoni, G., Paolucci, F., Solinas, P., Strambini, E. \& Giazotto, F. Metallic supercurrent field-effect transistor. Nat. Nanotechnol. 13, 802-805 (2018).

[18] Saito, Y., Nojima, T. \& Iwasa, Y. Highly crystalline 2d superconductors. Nat. Rev. Mater. 2, 16094 (2016).

[19] Caviglia, A. D. et al. Electric field control of the laalo3/srtio3 interface ground state. Nature 456, 624-627 (2008).

[20] Fatemi, V. et al. Electrically tunable low-density superconductivity in a monolayer topological insulator. Science 362, 926-929 (2018).

[21] Sajadi, E. et al. Gate-induced superconductivity in a monolayer topological insulator. Science 362, 922-925 (2018).

[22] Kononov, A. et al. One-dimensional edge transport in few-layer wte2. Nano Lett. 20, 4228-4233 (2020).

[23] Li, C., Guéron, S., Chepelianskii, A. \& Bouchiat, H. Full range of proximity effect probed with superconductor/graphene/superconductor junctions. Phys. Rev. B 94, 115405 (2016).

[24] Calado, V. E. et al. Ballistic josephson junctions in edge-contacted graphene. Nat. Nanotechnol. 10, 761-764 (2015).

[25] Xie, M. \& MacDonald, A. H. Weak-field hall resistivity and spin/valley flavor symmetry breaking in matbg. arXiv preprint arXiv:2010.07928 (2020).

[26] Mortensen, N. A., Flensberg, K. \& Jauho, A.-P. Angle dependence of andreev scattering at semiconductor--superconductor interfaces. Phys. Rev. B 59, 10176-10182 (1999).

[27] Bocquillon, E. et al. Gapless andreev bound states in the quantum spin hall insulator hgte. Nat. Nanotechnol. 12, 137-143 (2017).

[28] Moshe, M., Kogan, V. G. \& Mints, R. G. Edge-type josephson junctions in narrow thin-film strips. Phys. Rev. B 78, 020510 (2008).

[29] Uri, A. et al. Mapping the twist-angle disorder and landau levels in magic-angle graphene. Nature 581, 47-52 (2020).

[30] Kim, K. et al. van der Waals Heterostructures with High Accuracy Rotational Alignment. Nano Lett. 16, 1989-1995 (2016).

[31] Wang, L. et al. One-dimensional electrical contact to a two-dimensional material. Science 342, $614-617$ (2013).

[32] Orlando, T. P. \& Delin, K. A. Foundations of Applied Superconductivity (Addison Wesley, 1991).

[33] Russer, P. Influence of microwave radiation on current-voltage characteristic of superconducting weak links. J. Appl. Phys. 43, 2008-2010 (1972).

[34] Le Calvez, K. et al. Joule overheating poisons the fractional ac josephson effect in topological josephson junctions. Commun. Phys. 2, 4 (2019). 


\section{ACKNOWLEDGEMENTS}

We thank Benedikt Kratochwil, Peter Maerki and the staff of the ETH cleanroom facility FIRST for technical support, and Tobias Wolf for usefull discussions. We acknowledges support from the Graphene Flagship and from the European Union's Horizon 2020 research and innovation programme under grant agreement No 862660/QUANTUM E LEAPS. K.W. and T.T. acknowledge support from the Elemental Strategy Initiative conducted by the MEXT, Japan ,Grant Number JPMXP0112101001, JSPS KAKENHI Grant Number JP20H00354 and the CREST(JPMJCR15F3), JST. E.P acknowledges support from La Caixa Foundation.

\section{AUTHOR INFORMATION}

\section{Corresponding authors}

Correspondence and requests for materials should be addressed to Folkert K. de Vries (devriesf@phys.ethz.ch) and Peter Rickhaus (peterri@phys.ethz.ch).

\section{Author contributions}

E.P. made the stack and P.R. fabricated the device, with assistance from G.Z. T.T. and K.W. supplied the hBN crystals. F.K.d.V and E.P. performed the measurements. E.P. performed the simulations. T.I., K.E. and P.R. supervised the project. F.K.d.V., P.R. and E.P. wrote the manuscript with comments from all authors.

\section{Competing interests}

The authors declare no competing financial interests. 


\section{Supplementary Information}

\section{DEVICE}

a

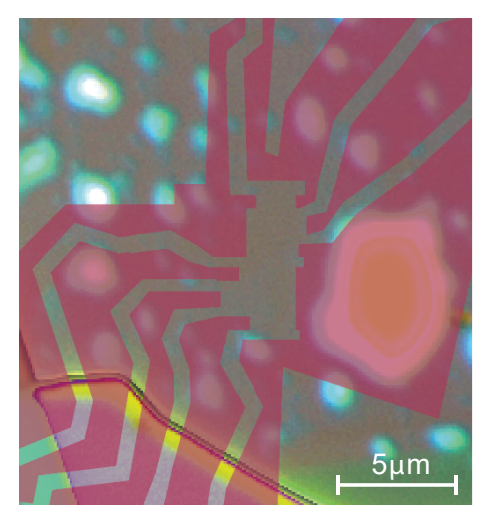

d

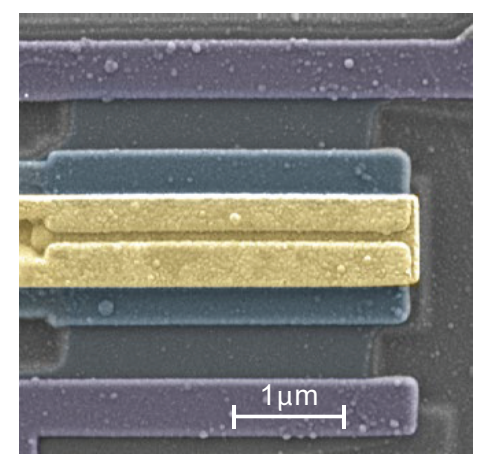

b
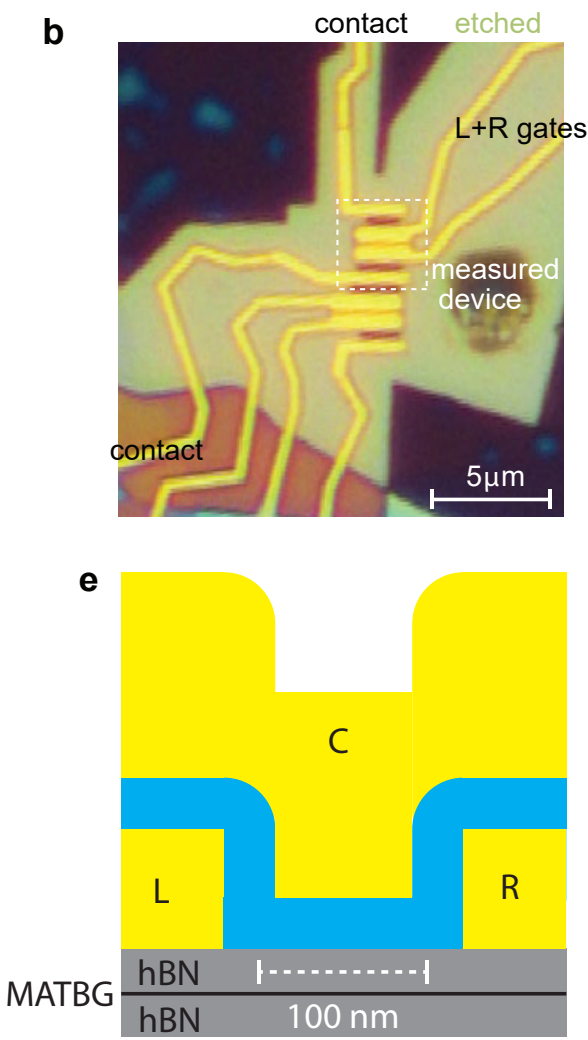

C

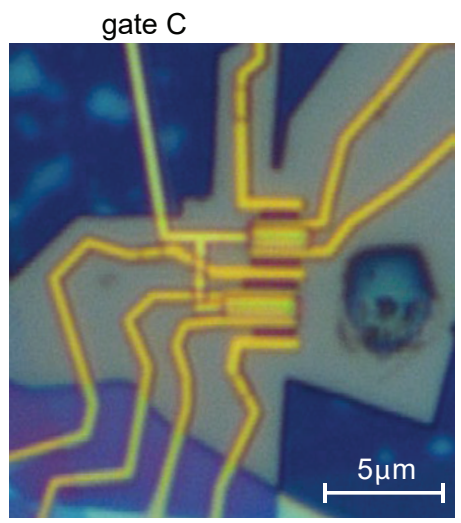

FIG. S1. Fabrication images of the device. a Optical image before fabrication, the red shaded area is the etch mask. $\mathbf{b}$ Optical image before and $\mathbf{c}$ after deposition of the central gate electrodes. $\mathbf{d}$ Scanning electron microscopy image of a similar device (not titled). e Schematic cross-section of the device, all layers are to scale.

In Fig. S1a-c we show optical images of the device fabrication process. The measured device is marked with a dashed square. Figure S1 13 shows that the device is placed carefully in-between the bubbles in the MATBG. The bubbles are expected to lead to twist angle inhomogeneity 29. In transport, we find that the lower device has a larger twist angle $\left(\theta=1.5^{\circ}\right)$ and does not show superconductivity. The narrow gap between the $\mathrm{L}+\mathrm{R}$ gates is well resolved in the SEM image in Fig. S1;, allowing us to identify a typical length of $100 \mathrm{~nm}$. The SEM image is taken from a similar device fabricated in the same batch and with similar geometry as the measured device (i.e. same length of $\mathrm{L}+\mathrm{R}$ gates and same gap between these gates in the design file). The schematic cross-section in Fig. S1p shows this length. Also, we estimate that the size of the gated region below gate $\mathrm{C}$ can vary by $\pm 10 \mathrm{~nm}$. 


\section{CRITICAL CURRENT DEPENDENCE ON TEMPERATURE AND MAGNETIC FIELD}

We measure $I_{\mathrm{c}}$ as a function of temperature $T$ and perpendicular magnetic field $B_{\perp}$ at $n=-1.53 \times 10^{12} \mathrm{~cm}^{-2}$, and present the result in in Fig. S2. The extracted critical magnetic field allows us to fit the Ginzburg-Landau equation $B_{\perp, c}=(\hbar / 2 e) / \xi^{2}\left(1-T / T_{\mathrm{c}}\right)$ for two-dimensional superconductors $\frac{13}{\text {, }}$, and obtain an estimate of the superconducting coherence length $\xi$.

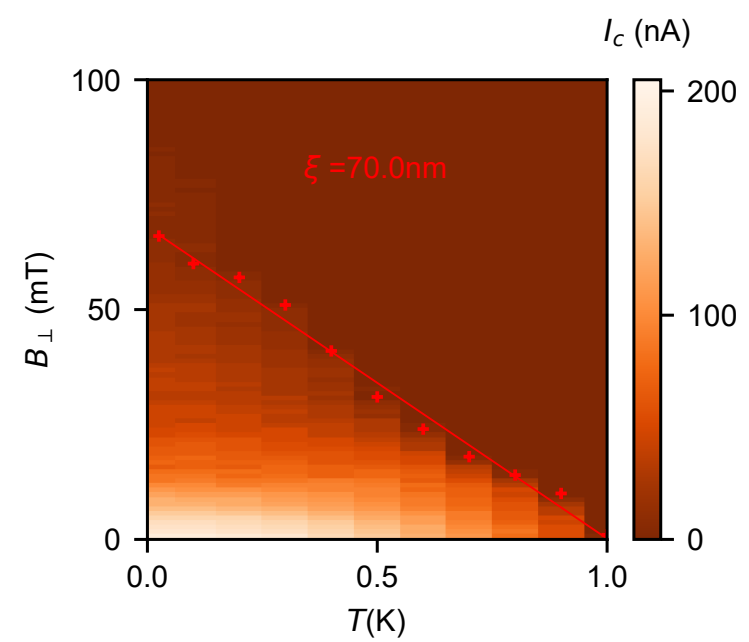

FIG. S2. $I_{\mathrm{c}}$ verus $B_{\perp}$ and $T$. The critical magnetic field is extracted and plotted as red crosses. A fit with the Ginzburg-Landau theory provides us with the estimate of the superconducting coherence length $\xi$ indicated. 


\section{FABRY-PÉROT OSCILLATIONS}

In addition to the step in $R$ observed in the dispersive bands, we investigate Fabry-Pérot resonances for the $100 \mathrm{~nm}$ JJ with $n_{\ell}=-1.5 \times 10^{12} \mathrm{~cm}^{-2}$, similar to earlier work in proximitized graphene JJs ${ }^{24}$. In Fig. S3a, b we show a zoomin to the region where $I_{\mathrm{s}}$ is modulated by $\sqrt{n_{\mathrm{j}}}$ for $n_{\mathrm{j}}<n_{\mathrm{s}}$. The conductance $G$ in Fig. S3k, recorded at $I=350 \mathrm{nA}$, behaves in a similar fashion. Local maximum values of $I_{\mathrm{c}}$ and $G$ occur if the Fabry-Perot condition is fulfilled. For constructive interference the condition reads $i \lambda=2 l$, where $i$ is an integer, $\lambda$ the charge carrier wavelength and $l$ the cavity length. The condition can be expressed as a function of density by using $\lambda=2 \pi / k$ and $k=\sqrt{(4 / g) n_{i} \pi}$, giving $n_{i}= \pm \pi i^{2} / l^{2}(4 / g)$. The third valence band is expected to be 4 -fold degenerate thus $g=4$. The observed maxima in $I_{\mathrm{c}}$ and $G$ (Fig. [S3b,c) are well captured by setting $l=75 \mathrm{~nm}$ (indicated by orange dotted lines) which is in good a agreement with $L_{\mathrm{j}}$.

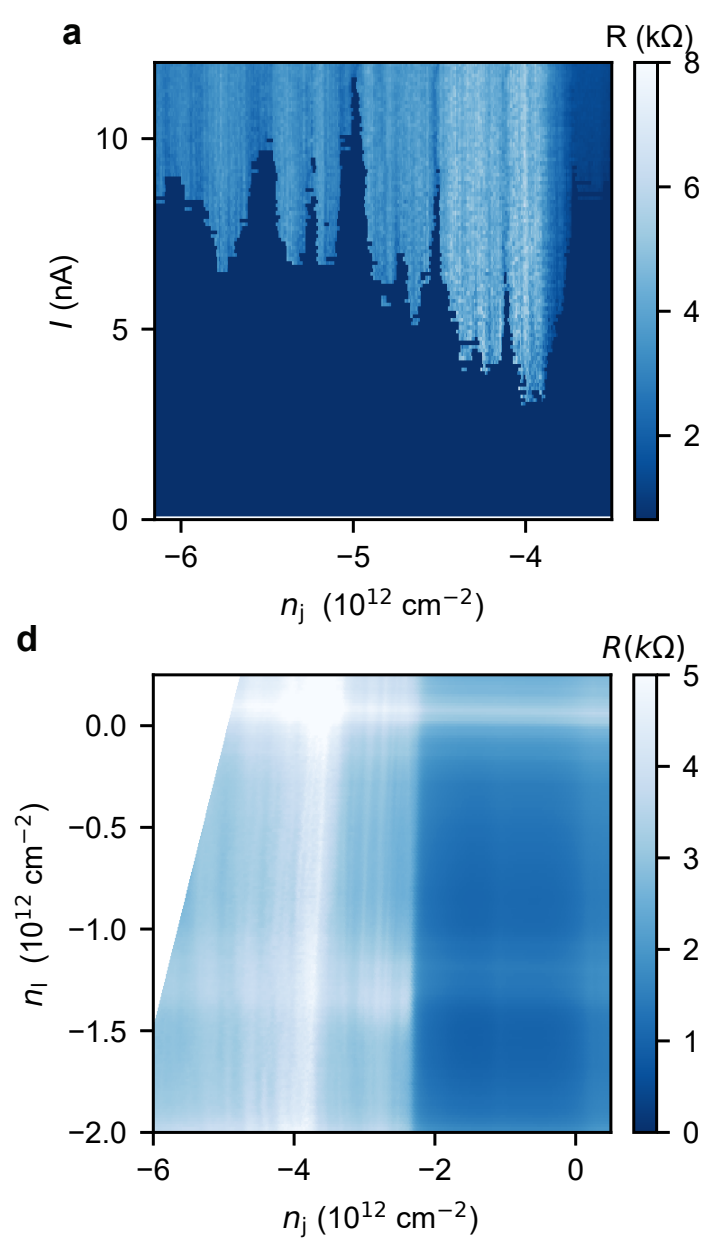

b

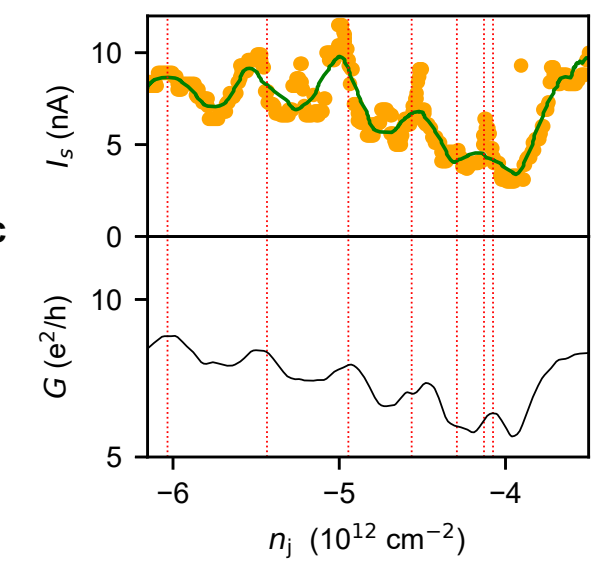

e

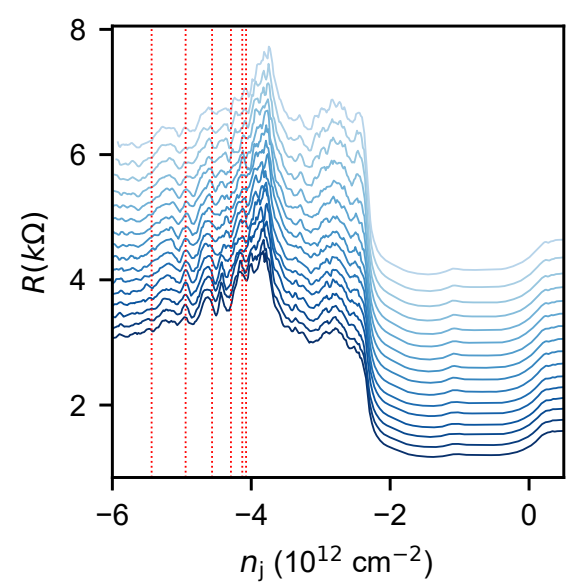

FIG. S3. Fabry-Pérot oscillations at the onset of the $3^{\text {rd }}$ moiré band. a Zoom in on $R$ versus $I$ and $n_{\mathrm{j}}$ for the JJ of $100 \mathrm{~nm}$ and $n_{\ell}=-1.5 \times 10^{12} \mathrm{~cm}^{-2}$. b $I_{\mathrm{s}}$ versus $n_{\mathrm{j}}$ extracted from a and a smoothed curve fit through it. c $G$ versus $n_{\mathrm{j}}$ calculated at $I=350 \mathrm{nA}$. d $R$ as a function of $n_{\ell}$ and $n_{\mathrm{j}}$, at $I=350 \mathrm{nA}$. e Extracted line-traces from (a). The orange dotted lines mark conditions for destructive Fabry-Pérot interference, assuming a cavity length of $75 \mathrm{~nm}$.

We further confirm the ballistic origin of the oscillations by investigating the dependence on $n_{\ell}$, see Fig. S3 d,e, where we plot $R\left(n_{\mathrm{j}}, n_{\ell}\right)$. The Fabry-Pérot oscillations show little dependence on $n_{\ell}$, confirming that the oscillations originate from a region below gate C. Such behavior becomes clear in the line traces $R\left(n_{\mathrm{j}}\right)$ for different $n_{\ell}$, see Fig. S33, where the $n_{\mathrm{j}}$ at which we expect constructive Fabry-Pérot oscillations are indicated by orange dotted lines. 


\section{SHAPIRO STEP LINE TRACES}

In Fig. S4 4 ,b we show line traces of the Shapiro-step spectra at both frequencies $5.1 \mathrm{GHz}$ and $1.8 \mathrm{GHz}$. We observe that the steps occur at the expected voltage following $\Delta V=h f_{\mathrm{rf}} / 2 e$.
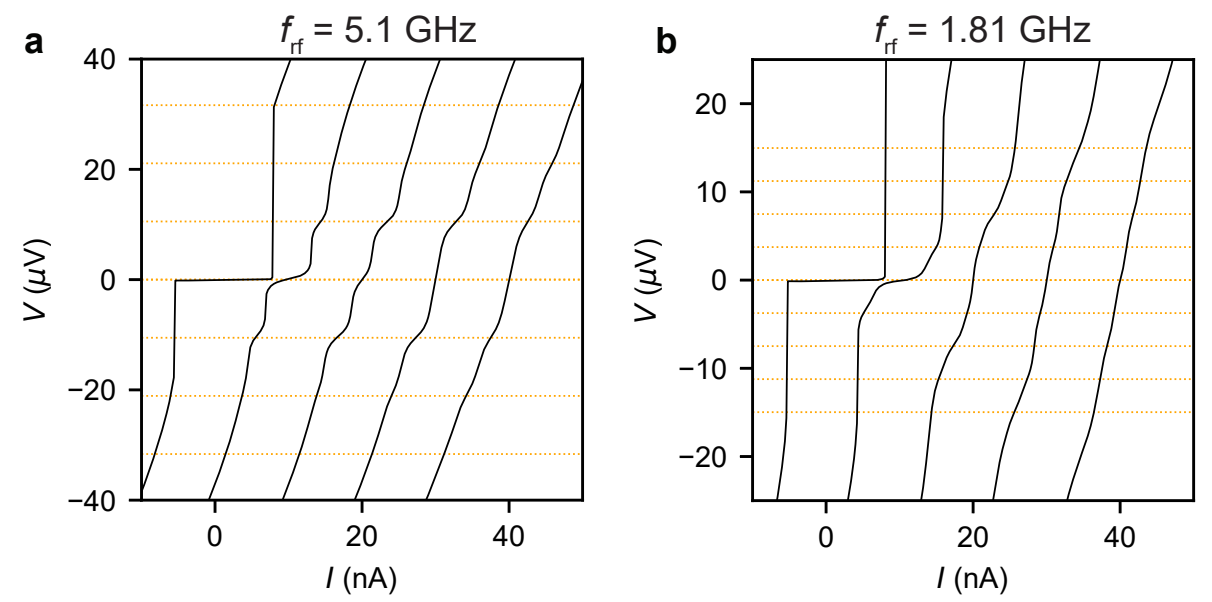

FIG. S4. Line traces of Shapiro steps at different frequencies. a,b $V$ versus $I$ show shapiro step line traces for the JJ of $100 \mathrm{~nm}$ with $n_{\mathrm{j}}=-4.6 \times 10^{12} \mathrm{~cm}^{-2} n_{\ell}=-1.5 \times 10^{12} \mathrm{~cm}^{-2}$ for the frequencies indicated. The traces are offset by $10 \mathrm{nA}$ and taken at $P_{\mathrm{rf}}^{1 / 2}=0,0.015,0.022,0.028,0.035$ for $\mathbf{a}$, and $P_{\mathrm{rf}}^{1 / 2}=0,0.005,0.009,0.014,0.019$. The orange dashed lines mark the expected steps at multiples of $\Delta V=h f_{\mathrm{rf}} / 2 e$. 